\title{
Measuring Stability and Change: Methodological Issues in Quality of Life studies
}

\author{
Filomena Maggino ${ }^{1} \cdot$ Carolina Facioni $^{2}$
}

Accepted: 30 September 2015

(C) Springer Science+Business Media Dordrecht 2015

\begin{abstract}
The aim of the paper is to show how the variety of approaches to study social change may result in a challenging complexity for the social scientist, starting from the difficulty of defining the concept of "change" itself and managing it through observed data. This is particularly true in presence of complex phenomena, such as those defining and composing the quality of life. What should be pointed out is that quality of life studies not only are focused on the present time but have also long term perspectives. This represents the link between studies on quality of life and forecasting. When applied to the field of quality of life, the typical logical approach to forecasts, based upon inferential statistics, could reveal its limits. Those limits are related to different aspects: $e$.g., the forms of relationships between different aspects of the phenomenon, which can be linear and nonlinear; the dimensionality of phenomenon, which can turn out to be very complex; the causality, which could be direct or indirect; the entity of change, which implies the idea that also small change can have great impact; the perspective of observation, which can be internal or external and local or global. Consequently, the study of change related to quality of life needs, in addition to the traditional statistical tools as well as the tradition of social indicators, a different approach. Although the Futures Studies are not a proper science, nevertheless their approach to social research may ensure the requested accuracy of a scientific forecasting process.
\end{abstract}

Keywords Conceptual approaches in forecasting $\cdot$ Measuring change $\cdot$ Futures studies

Carolina Facioni

facioni@istat.it

Filomena Maggino

filomena.maggino@unifi.it

1 Department of Statistics, Informatics, Applications "G.Parenti" (DiSIA), University of Florence, Florence, Italy

2 National Institute of Statistics - ISTAT, Rome, Italy 


\section{Introduction}

Dealing with trends in quality of life can have different aims and interests. One of them is related to the possibility of designing possible future states.

In statistics, this exercise is not new and belongs to the field of inferential statistics aimed at establishing knowledge from data by taking into account the error associated to them. This kind of knowledge allows statistical forecasts and predictions to be determined.

One of the instrumental concepts and rational allowing trends to be read is that of "change" (and, of course, its opposite meaning, "stability"), which is far from being easy to be defined and managed through observed data. This is particularly true in the presence of complex phenomena, such as those defining and composing the quality-of-life. That is because the logical approach underlying inferential statistics could reveal its limits. Those limits are related to different aspects, such as (1) forms of relationships between different aspects of the phenomenon, which can be linear and non-linear; (2) dimensionality of phenomenon, which can turn out to be very complex; (3) causality, which could be direct or indirect; (4) entity of change, which implies the idea that also small change can have great impact; (5) the perspective of observation, which can be internal or external and local or global.

In order to overcome those limits, a different approach is needed, able to manage the concept of change and its observation, in the perspective of picturing and sketching the future.

The different approach should be also related to the idea that the forecast of an observed phenomenon can be defined by more than one "future". In other words, forecasts based upon observation of changes are something related to decision and not to something deterministic.

In the first paragraph, we retrace the conceptual perspectives used in the statistical forecasting exercise. In the second, how the concept of "change" is managed in statistical and methodological terms. In the third, an alternative approach (named "future studies") to analyzing change in order to sketching possible futures is introduced and illustrated.

The present paper has to be considered as a collective work. Although, paragraph 1 and 2 have to be ascribed to Filomena Maggino, while paragraph 3 to Carolina Facioni. The opinions expressed by Carolina Facioni should be related only to her and do not necessarily reflect those of the Italian National Institute of Statistics.

\section{Conceptual Perspectives of Forecasts}

What is always stressed in any discussion around predictions is that they are related to the level of understanding of phenomena. This means that a close relationship exists between forecasts and explanations.

\subsection{Forecasts and Explanations}

According to the classical inductive approach, which tries to relate consecutively aspects such as changes, explanations and inferences, finding causes represents the sufficient/ necessary condition for explanation. This requires an experimental design which is impracticable in the field of quality-of-life research. The difficulties in inductive explanations concern the time dimension (not always is possible to observe a clear sequence of 
events), the actual impossibility to identify the set of all causes of any given event, the possible existence of circular relationships among events, and, last but not least, the indeterminacy (uncertainty: when two phenomena cannot be measured in the same moment are incompatible).

According to the deductive approach, there is no time parameter. Actually explanations and forecasts are different only with respect the time: explanation concerns past and present events, forecast concerns the future. In both situations, the conclusions are valid only if they are included in a conceptual and theoretic framework.

The probabilistic explanations require, on one hand, specific statistical data and, on the other hand, probabilistic laws. ${ }^{1}$

However, explanations deducted from causal relationships show some problems since it is not [always] possible to (1) understand which are the relevant information necessary for explanations, and (2) to establish a certain relationship between causes and effects; in other words, different logical (causal) order can be ascribed to the phenomena and can lead to different explanations (in other words.

Other approaches include the subjective model of explanation (De Finetti), according to which explanations are related to several aspects such as the owned and shared background information and the subject's expectations.

\section{A Logical Perspective Supporting Prediction: Observing Change}

In studying social phenomena, such as wellbeing, one of the more interesting issues to be encountered, considered and managed is studying dynamics, expressed in terms of stability and change.

Generally, studying and describing detailed patterns of change allow (Goldstein 1979; Menard 1991) obtaining insight into underlying causal processes (Engel and Reinecke 1996) and predicting change values from those obtained in the past.

A consistent study requires two aspects to be carefully and systematically considered:

1. Theoretical, concerning the definition of change and the identification of modifiable dimensions or dimensions that may significantly change

2. Methodological, concerning the identification of adequate and proper

1. Study designs (e.g., differences between changes observed at micro level, individual/subjective change, and at macro level)

\footnotetext{
${ }^{1}$ Statistics deal with-among many issues-identifying regularities in collected information (data). Those regularities can be observed in terms of tendencies when a certain phenomenon is observed in the course of time, or in terms of relationships, when regular convergences or divergences are observed among phenomena.

Observing regularities allows interpretations and explanations to be hypothesized. Consistently with the acquired knowledge, which can be formalized in a model, it is possible to speculate about how the phenomenon will turn up in the future. In other words, in the statistical context, models allow forecasts (projections) to be formulated also in the perspective of taking decisions (which could influence trends of certain events).

In statistical model definition, three main approaches can be identified, (1) from observing a part (estimation), (2) from defining a hypothesis (testing through falsifiability), and (3) from observing the past (forecasts).

Applying such approaches lead to a statistical decision which is always expressed in probabilistic terms. In other words, the statistical forecast always contains an uncertainty component, expressed in terms of probability.
} 
2. Measuring procedures (in terms of reliability and validity)

3. Data analysis approaches (in terms of methods and techniques).

With reference to these aspects, four fundamental validity issues can be observed (Campbell 1963; Visser 1985):

(a) Internal validity, concerning the possibility to come to correct conclusion within a particular study (basic logic of the whole study),

(b) External validity, concerning the possibility to make correct generalizations (sampling design),

(c) Construct validity, concerning the representativeness of observation as regards what was supposed at theoretical level (measurement procedure),

(d) Statistical conclusion validity, concerning correct treatment of data in order to study variations in observed data (data analysis approaches).

Such items correspond to the crucial points that have to be defined and handled in studies based on change (Bryk and Raudenbush 1992).

\subsection{Theoretical Aspects}

Investigating phenomena under the perspective of change requests the definition of a model able to describe the change. According to different scientific fields (economics, behavioral sciences, and so on), different kinds of models have been identified.

When the description is based on mathematical definitions (Menard 1991; Brown 1995), the models can be (Fig. 1):

- Deterministic, when the change is conceptualized by fixed patterns or laws, defining whether, how and how much, according to some hypotheses, a variable changes as a response to change in other variables (individuals/groups change models); the statistical approach is instrumental. The deterministic approaches:

- Lead to fractions or events,

- Are more flexible in nonlinear specification, allowing a more realistic understanding of the complexity of [social] dynamics,

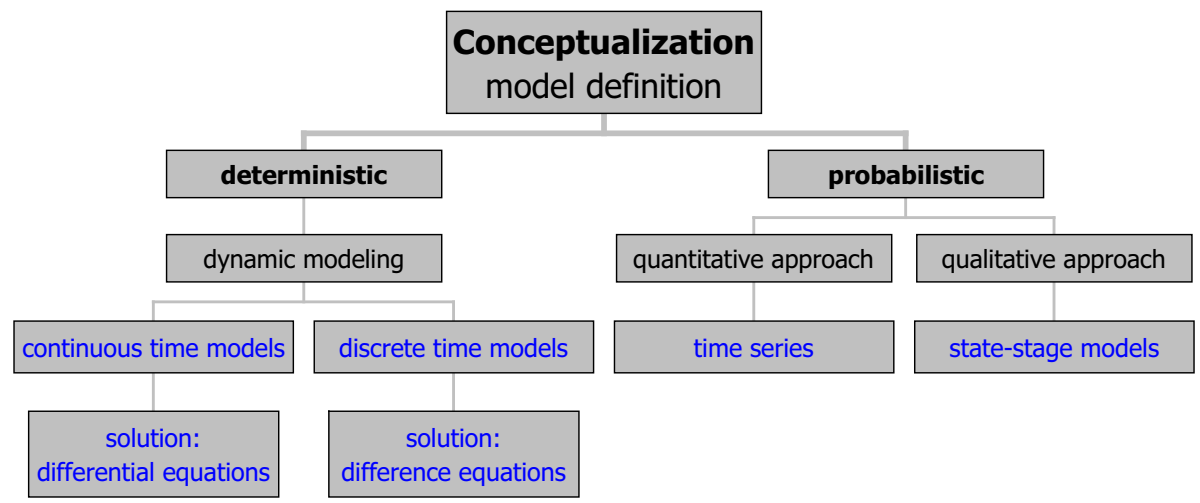

Fig. 1 Characteristics of models of change 
- Need a series of statistical measures evaluating the model (measures of fit, tests of the significance of the parameters, measures of relative importance of the parameters, etc.).

- Probabilistic or statistical, when the goal is to predict with some accuracy, at group level, the proportion/percentage of cases that will change, the proportion/percentage of cases that will change in a certain direction, the average amount by which they will change; the underlying assumption allowing the probabilistic description is that there is some influences on a process defined probabilistic at individual level. Probabilistic approaches:

- Yield probabilities of complete events,

- Are based upon standard statistical methods, such as ordinary least squares (OLS) ${ }^{2}$, and estimation, such as maximum likelihood approach,

- Allow measurement error to be included in the model.

Both deterministic and probabilistic models can be distinguished with reference to time:

(a) Deterministic continuous time models,

(b) Deterministic discrete time models,

(c) Probabilistic continuous time models (in which the process requires the assignment of a probability to an event in an infinitesimal period of time),

(d) Probabilistic discrete time models (in which the process, generally defined by generational occurrences, requires the separated measurements related to significant time sections).

The choice between continuous and discrete approaches depends upon measurement opportunities. Generally, and not only in social studies, the measurement of phenomena occurs at discrete points of time, more or less regular, regardless of the dynamic structure (Brown 1995).

Models with randomly fluctuating parameters can also be identified, applicable especially when the parameters are functions of other variables containing stochastic components (Brown 1995).

\subsubsection{Deterministic Models}

Deterministic models, defined in terms of quantitative change, express values of the changing variable as a function of time. The mathematical formula/equation can be defined according to two goals (Menard 1991):

- Description of change: the formula includes only variable and time

- Explanation of change: the equation involves the introduction of other variables.

The most useful approach to represent processes of change over time, considering time an explanatory dimension, is dynamic modeling, whose main objective is 'to understand, substantively, the mechanisms that are generating change in some observable phenomenon, and then to translate this set of ideas into mathematical language' (Huckfeldt et al. 1982). In other words, the objective is to make an adequate synthesis of observed measures in terms of a small number of parameters.

${ }^{2}$ Notice that also ordinary least squares method is based on a deterministic formulation for an equation defining a line (Brown 1995). 
The application of dynamic modeling is based mainly upon functional/differential models (Visser 1985; Menard 1991; Huckfeldt et al. 1982) used principally as theoreticaland not analytical-tools; they help in considering the possible relations between unobservable variables. The application of this approach in social sciences is essentially theoretical. In fact, the practical use of these models is often obstructed by difficulties in specifying the process in detail, as requested, and by treating time variable as a continuous variable. In this perspective, difference equation models, considering time as a discrete variable, may represent a solution.

Subsequently, according to the two different treatments of time dimension (central in dynamic modeling) we can distinguish between two different approaches.

- Deterministic models for continuous time treatment. These models define growth dynamic processes, assuming infinitely small time units and instantaneous rates of change. They are mathematically expressed in terms of differential equations (Visser 1985; Menard 1991; Huckfeldt et al. 1982). An example of deterministic model expressed in terms of differential equations is the internal-influence diffusion model. It concentrates interest on the study of innovation diffusion and expresses this as a function of time (cumulative numbers of adopters of an innovation at a given point of time):

- Deterministic models for discrete time treatment. In these models, the period between successive events is usually fixed according to calendar, simplifying the identification of time. They are mathematically expressed in terms of difference equations, (Huckfeldt et al. 1982) for which time is treated as a series of discrete, equally spaced units. This approach can define model as a combination of single/interdependent and linear/nonlinear equations. It seems to be more applicable in many social studies since allows management of:

(a) Observations occurring in discrete time,

(b) Great diversity patterns of qualitative behavior,

(c) Models without requiring high level of mathematical formalizations.

\section{Theories related to deterministic models}

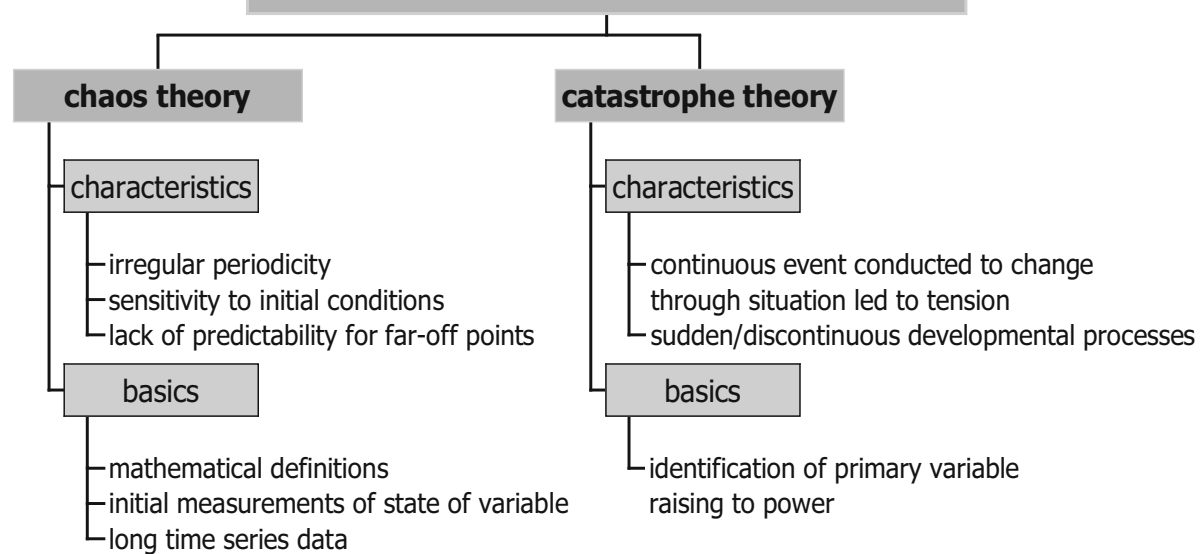

Fig. 2 Characteristics and basics of theories related to deterministic models 
In certain cases, it is possible to assume that observed discrete events are manifestations of an underlying continuous process or that the continuous process manifests itself at discrete points of time. However such approaches are applicable (Huckfeldt et al. 1982) only to change produced by a fixed dynamic structure (synchronic change). Treatment of process of moving from one structure into another (diachronic change) requires different approaches.

Among theories supporting deterministic models, two deserve a citation, for the possible applications that they may have social studies (Fig. 2).

- Chaos theory (Brown 1995). Chaos is an irregular oscillatory process that can be observed in human behavior as well. Many repetitive behaviors can be described in terms of oscillatory process (long and short cycles) both in micro (daily cycles of everyday life) and macro (cyclic events like periodic vote) processes. Repetitions are not always regular with regard to time. Consequences and effects of irregularities can be considered positive (as in some characteristics of creativity) or negative (irregular rest or food consumption cycles).

A chaotic system is identified by the following fundamental characteristics:

(a) Irregular periodicity (absence of a repeated pattern),

(b) Sensitivity to initial conditions (small changes in the initial conditions in a chaotic system produce dramatically different evolutionary processes),

(c) Lack of predictability (sensitivity to initial conditions makes chaotic system unpredictable; however prediction is possible when the interest is concentrated on the movement between two relatively close points on a trajectory).

Requirements of chaos modeling are

- Dimensionality (minimum of three independent variables in continuous time: differential equations; one independent variable in discrete time: difference equations $^{3}$ );

- Nonlinear models (at least one term in one equation must be nonlinear).

One of the approaches aimed at estimating nonlinear interdependent systems potentially chaotic is nonlinear least squares.

The manageability of chaotic system, presenting these characteristics, relies on

- Numeric calculations,

- Initial measurements of the state of variable,

- Long, and sufficiently close, time series data. ${ }^{4}$

These make chaotic processes difficult to be dealt with, since chaotic systems can be most likely affected by combinations of computational and measurement errors at original data level.

The apparently restrictive requirements do not imply rare chaotic systems in the real world, especially in social system.

- Catastrophe theory (Brown 1995). Catastrophe theory is based upon the concept of bifurcation, which is an event that occurs in the evolution of a dynamic system in which

\footnotetext{
3 The term 'map' in place of 'function' is usually applied with reference to difference equations that associate paired data for discrete time. The general form for these functions is logistic.

4 The concept of close points of time in a data series refers to time variability of phenomena under study, especially in social research.
} 
the characteristic behavior is transformed (Brown 1995). Models based on catastrophe theory allow analysts to describe a discontinuous phenomenon controlled by continuous variables through graphs in $k$-dimensional spaces. ${ }^{5}$ They are limited in the treatment of processes characterized by sudden change or discontinuous development. They describe a process that can be conducted to a generalized change through a situation led to tension. This requires the identification of a primary variable raising to some power. One of the approaches supporting the estimation of catastrophe processes is the nonlinear least squares method. Different measures of fit are calculated for "onecase many time points" data and for "many-case few time points" (also two points). (Brown 1995)

In order to develop a meaningful catastrophe model, social theory perspective is greatly usable; algebraic forms are only helping instruments in building models. (Brown 1995)

Also catastrophic systems are not rare in the real world even if data applications are rarely found. Among the most well-known applications (cusp catastrophe) of this theory in studying behavioral processes concerned ethological (aggressiveness) and social (prison riots) studies and date back to René Thom e Christopher Zeeman (Visser 1985).

\subsubsection{Probabilistic Models}

Probabilistic models allow measures made at one occasion and measures made at the successive occasion to be directly related. In many case this approach assumes causal relationships between measurements. The adoption of probabilistic models requires implicit recognition of time direction (measurements at one occasion are dependent on measurements at earlier occasions). Pre-post models can be considered part of these models (Visser 1985).

Figure 3 summarizes the main characteristics of the two probabilistic model approaches: quantitative and qualitative.

Quantitative Approaches The typical quantitative descriptive approach defined in the ambit of probabilistic models is represented by the time-series approach. It allows small numbers of cases (typical one) on a large number of periods of time to be described. The description (Visser 1985) of time-ordered data is carried out in order to distinguish four processes:

(a) Noise/random process (probabilistic component, present in all stochastic models),

(b) Autoregressive $(A R)$ process (present values of a variable depending on past values at some specific lag/s or interval/s),

(c) Moving average (MA) process (past value of the noise influencing present values)

(d) Integrated $(I)$ process (measurable trend over time in the values of the modeled variable, but in which there is no trend in the series detectable by subtracting values of the variable from values of the variable at later time).

Models, quantitatively expressed, always involve noise component and may incorporate one, two or all three other components. The objective is to define the over time change of variables measured in terms of a stationary time-series.

\footnotetext{
5 The study of qualitative differences between classes of graphs connected by continuous transformations is part of topology.
} 


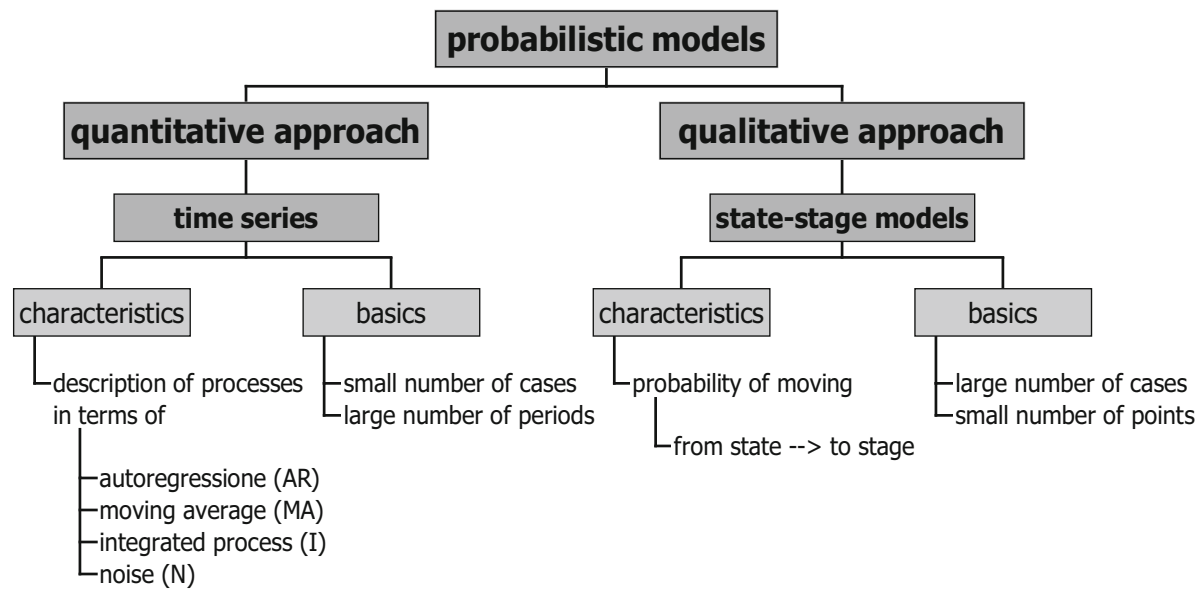

Fig. 3 Characteristics and basics of probabilistic models

Qualitative Approaches Typical probabilistic change models defined in qualitative terms (categorized classifications) are stage-state models (or dynamic typologies), in which movements of subjects from one category to another over time are modeled (Menard 1991). In particular, stage-state models are concerned with the probability of moving from one value (state) to another value of a variable by a given period (stage). For multiple category variables, separate probabilities of transition (movement from one value to another in a given interval between periods) are calculated for each pair of origin-destination states (state at, respectively, the beginning and the end of the interval). When origin and destination states are the same, the transition probabilities indicate the stability in the state over time. Stage-state models can be described by using (Menard 1991):

(a) Simple transition matrices, with no assumption about underlying properties of the transition matrices (based on measurement taken for few or two, periods),

(b) Markov models, including Markov chains (based on measurement taken for few or two, periods),

(c) Log-linear models (based on measurement taken for few or two, periods),

(d) Univariate life table models (based on measurements taken for several periods),

(e) Univariate survival models (based on measurements taken for several periods); they assume that survival rate follows fixed distribution these models are useful to model processes like recidivism, labor force participation, marital history events and other transition events among discrete states.

The $a$ and $b$ models are based on simple row percentages from cross-tabulations or contingency tables that compare the values of a variable for the same set of cases.

For Markov and life table models it is possible to define a particular state that, once entered, cannot be removed (absorbing state). ${ }^{6}$ In these cases, it is possible to calculate (Menard 1991):

- The proportion of cases at absorbing state (and at each other state) at a given period;

6 A typical example of absorbing state is death. 
- How long it will take all cases, or a certain proportion of cases, to enter the absorbing state.

Some stage-state models were developed in socio-economic field with reference to particular concepts.

- Models connected to the concept of 'wastage' (or 'turnover' or 'attrition'). This model is concerned with the loss (and its dynamics) of individuals in a closed system (e.g. a firm), and is dealing with a stochastic process, developing over time. The development of the model allows a particular system to be compared over two different periods; many functions may be described, like parameter of the propensity-to-leave function (Bartholomew 1996).

- Models connected to the concept of 'mobility'. This concept is typical of social researches concerning social or occupational mobility (Bartholomew 1996). Particularly, in social studies inter-generational mobility, definable in terms of transitions of family lines from one generation to the next, and intra-generational mobility, definable in terms of changes of class within the lifespan of the individual, can be defined. The mobility process can be observed also in other fields (movement of firms, geographic mobility of individuals, families, and so on). Each mobility model is defined by two dimensions:

(a) Classes, between which movement takes place; they can be formed in a variety of ways (income, occupation status, occupational skill, satisfaction level, etc.);

(b) Time, that generally is treated as discrete, since usually data are available at fixed intervals of time (months, year, contracts of employment, holiday periods, and so on).

Consequently, definition and analysis of mobility models refer to discrete classes changing state at fixed time intervals.

Approaches to modeling are related to two different aspects of mobility processes:

- Pure mobility: the number of individuals (such as fathers) in each class are treated as fixed while the number of lines moving to other classes (such as sons) are modeled;

- Structural mobility: the number of places of individuals in the second occasion (sons) are treated as fixed while the reverse flows of vacancies back to individuals of the first occasion (fathers) are modeled.

Both processes depend upon the discrete time Markov chain (Bartholomew 1996). Alternative approaches to discrete change modeling are based on log-linear models and latent class models (Lazarsfeld and Henry 1968; Bartholomew and Knott 1999). Wiggins and Coleman models are considered extensions of Markovian models incorporating error of measurements (Markus 1979).

\subsubsection{Applications in Quality-of-Life Studies}

Generally, the approaches reported here found applications in different studies, like those concerning subjective characteristics (with reference to sociopolitical attitudes and behavior) and may find applications in subjective quality of life studies as well.

With reference to deterministic approaches, past applications of chaos theory to social setting were made possible only by simulations because of lack of adequate measured data. Nowadays, availability of organized and systematic data (individual and aggregated) could 


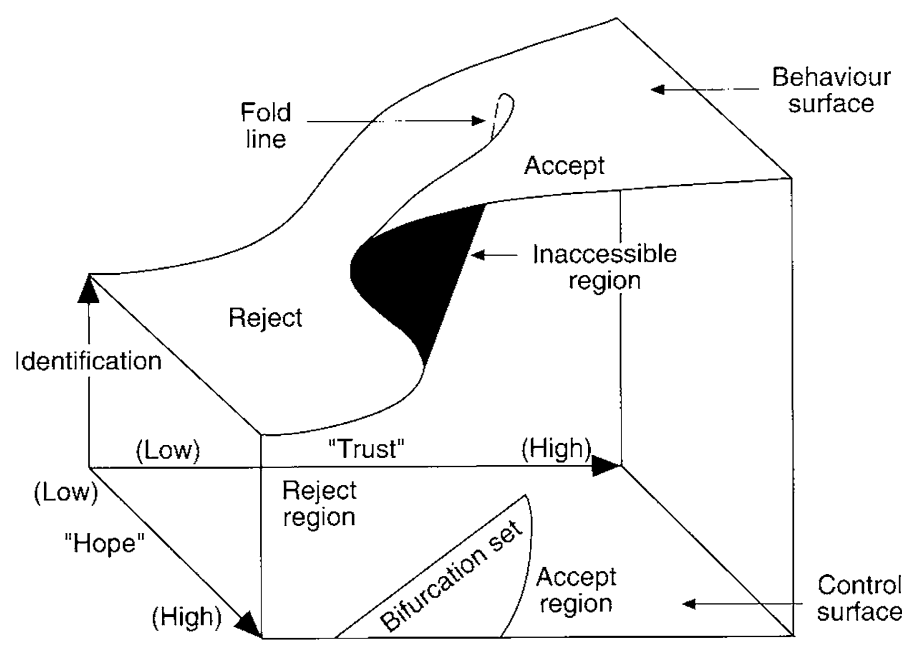

Fig. 4 The graphical representation of an application of cusp catastrophe theory to social cohesion dimensions

allow evidence of chaotic models in social scenery to be found, especially for those social phenomena having long and periodic properties, as it happens in many models concerning individual behaviours (Brown 1995), also related to subjective quality of life ambit.

Possible applications of catastrophe theory to quality of life study were seriously limited because of the complicated structures of models and the technical problems in defining social processes; in other words, catastrophe theory remains an interesting but not applicable model proposal (Visser 1985). An interesting application concerns the relationship between hope, trust and identity, which can help in disentangling the citizens' living conditions during the crisis (Fig. 4).

Among the probabilistic time-related approaches, models are usually expressed in terms of growth (growth/development/achievement models) and found applications in achievement research and in many approaches to educational and psychology studies (Rogosa et al. 1982; Rogosa and Willett 1985; Embretson 1994) (Fig. 5).

The main advantage in using growth models is connected with the possibility of predicting values of the variable (Goldstein 1979), by identifying patterns, linear and not linear, of events or relationship. ${ }^{7}$ However, the main limit of the approach is related to the conceptualization of change only in terms of gains, of a 'step by step' function in analogy to a 'building block model', where no negative blocks are defined. Such model may fail in describing processes underlying well-being dimensions. This is because the adoption of growth models is not always justified, especially in studying well-being processes, where models of change cannot be defined only in terms of time related growth; in fact, differences in both directions are expected depending on different predictive factors (for examples we may expect that positive and negative life events may yield individual change

7 In statistical terms, this requires (Goldstein 1979; Firebaugh 1997; Bryk and Raudenbush 1992):

- Identifying an interpolating line, connecting points for the observed group (individual change model),

- Estimating model parameters (slope and intercept) in order to identify a general modification of characteristic and to analyze differences among individuals (general change model). 


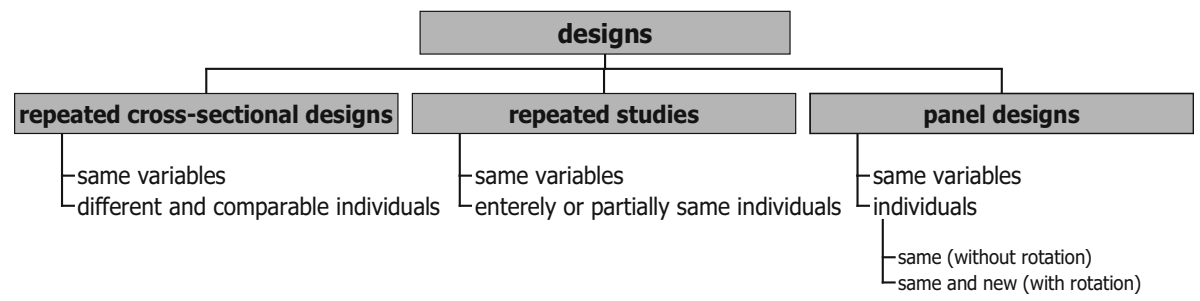

Fig. 5 Characteristics of typical designs

in different directions on the same dimension). Moreover, growth-models parameters are directly comparable between different cases (subjects, nations, organizations, and so on) only after testing comparability of the cases' starting conditions.

Modeling changes in wellbeing through differences (change/mobility/residual) models seems to be more interesting since this approach may help to explore presence of causal patterns.

\subsection{Methodological Aspects}

Measuring and analyzing change require some methodological issues to disentangle.

In particular, the solutions should be adequate to the level of observation, which for quality of life is typically micro, in other words, the observation is accomplished at individual level, while the analysis is generally performed in order to obtain results and interpretation at both macro and micro level.

The methodological issues related to observation concern the design and the measurement.

\subsubsection{Design}

Studying well-being dynamics requires the definition of designs aimed at obtaining measures which can be analyzed in terms of change at individual level with respect to specific dimensions. Any design is characterized by two features:

- Approaches aimed at collecting data for each variable for each point of time,

- Individuals involved from one occasion to the following (the same, comparable at individual level, comparable at group level, and so on).

According to these, different macro typologies of design (Goldstein 1979; Menard 1991; Firebaugh 1997) can be identified (Fig. 4).

Repeated cross-sectional designs produce data observed on different but comparable individuals on same variables at each occasion. In some circumstances, one-occasion cross-sectional survey design can be applied also in change studies (Hagenaars 1990); these can be done by:

- Using information, provided by respondents, about their past behaviors, attitudes, beliefs, etc.;

- Comparing different age groups in order to observe generational change (meeting two conditions: the differences observed at one occasion can be interpreted as a difference between generations; the generational characteristics are stable over time); 


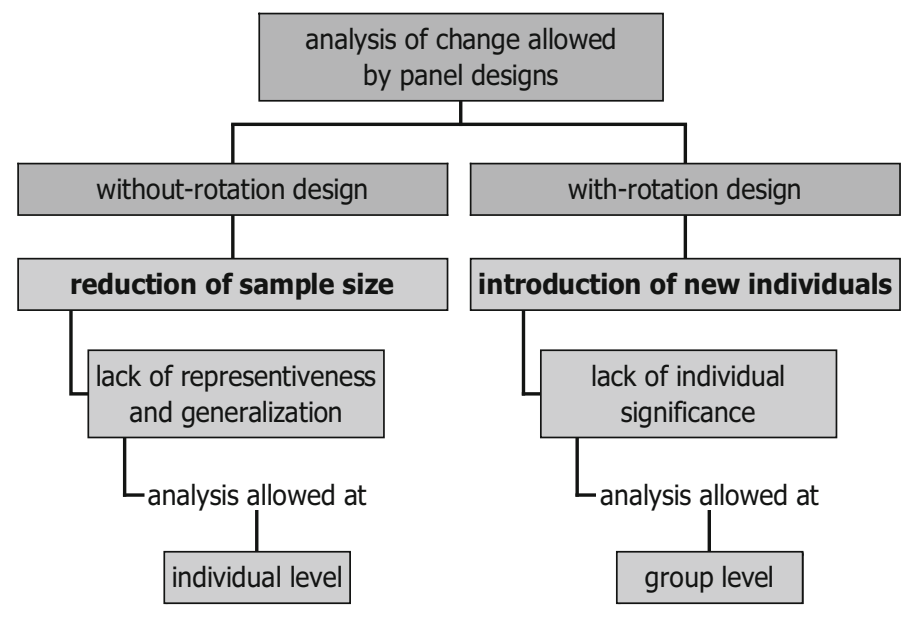

Fig. 6 Different analysis approaches allowed by panel designs

- Interpreting cross-sectional relations between certain variables as explanation of change over time (education level and kind of work); this can be done when one condition is meeting: the system of variable under study is in a dynamic equilibrium.

In spite of interesting application in change studies, in general this design should be carefully considered in terms of reliable and valid evidences (Hagenaars 1990).

Repeated (or "periodical" or "recurrent") designs involve:

- Groups entirely or partially formed by same individuals

- Groups formed by different individuals.

Groups identified for repeated studies may not keep representativeness as regard to reference population.

Panel studies can be defined in two different ways:

- Without rotation: repeated surveys on same individuals. The goal is to study the change at individual level. Reduction of sample size does not allow outcomes to be generalized

- With rotation: repeated surveys on same individuals as well as new ones in order to maintain statistical generalization of sampling outcomes.

The two approaches allow respectively two different levels of change analysis (Fig. 6) both useful in quality of life studies.

Panel studies are defined by the designed number of points of time, generally named waves. The number of waves depends on the defined model. Usually, studies dealing with time related models require longitudinal design with more than two waves (Goldstein 1979). On the other hand, difference models can be significantly based also upon two waves. ${ }^{8}$

\footnotetext{
${ }^{8}$ As reported below, the combination of waves and number of involved variables defines different kind of model; the simplest one is the two-wave two-variable $(2 \mathrm{~W} 2 \mathrm{~V})$ model.

9 Many interesting works analyze data obtained by repeated designs in order to describe national/regional trends.
} 
Repeated measurements designs may also be distinguished in (Lindsey 1999):

- Prospective design, in which data are collected at two or more distinct occasions on same individuals and variables; typically, this approach concerns survey designs (panel and cohort designs) and experimental designs (clinical trial)

- Retrospective design, in which values of previous explanatory variables, concerning past occasions, are collected and investigated for each respondents. This design is typical of experimental study (case-control study in medical sciences) and is difficult to be applied in social studies.

In well-being studies, repeated survey designs seem to be most applicable especially in comparing data at aggregated levels. ${ }^{9}$ On the other hand, large panel designs are recommended in order to obtain more explicative information about individual processes about subjective change in well-being dimensions.

\subsubsection{Measurement}

In practice, measurement of change requests at least

(a) Two measures obtained in two different moments on same the same unit or

(b) One measure obtained in one different moments for each of two comparable units.

In case $a$, change is measured at individual level, in case b, at group level. In both cases measurement of change requires, as a rule, a consistent operational definition of change based upon the definition of:

1. Methods for measuring amount of change (operational definition of change),

2. Requirements for comparability conditions,

3. Approaches for testing reliability and validity of measures over time.

Comparability Measuring change should meet conditions of comparability concerning the (1) studied characteristics, (2) subjects, (3) data-collection procedures, (4) data-collection instruments, and (5) measurement approaches.

Studied characteristics: comparability of characteristics over time has to consider their eventual tendency to be time-related, age-related or event-related; in each case, the observed change may lead to different interpretations. Consequently, in some studies, in order to maintain comparability of characteristics at group level and/or individual level, different instruments for different conditions (in terms of point of time, of individual age, of survey condition, and so) have to be applied (Goldstein 1979).

Subjects: individuals should be comparable with themselves; frequently even if comparable instruments are available, subjects may result incomparable (e.g., change in level of understanding).

Data-collection procedures: adopted data-collection techniques (e.g., paper-, CATI-, web-questionnaire) should be comparable from one occasion to the other; this condition is difficult to be completely controlled; for example, the interviewers may be different from one occasion to the other, each interviewer may use different approach from one occasion to the other. In order to avoid introducing measurement errors yielded by interviewers (e.g., in classification or/and in coding), careful training and detailed instructions should be considered and adopted.

Data-collection instruments: the adopted instruments can be (Webster and Bereiter 1963): 
- Identical: they have identically worded items presented in the same order and the same format to the same/comparable person;

- Matched: they have items matched through statistical criterion; in this perspective, identical instruments represent a special case of matched ones;

- Not-matched or randomly matched instruments.

The presence of other factors (events occurring between the two occasions, memory, learning, survey different conditions, change in characteristic definition, and so on) could prevent from meeting comparability of instruments and make impracticable the use and application of the same instrument.

Measurement approaches concern (1) level of measurement, (2) type of measure and (3) nature of data.

Level of measurement: in this case the comparability regards

- The scaling technique: the comparability has to consider that each technique is defined by scale reference (evaluation, preference, perception, image, judgment), scale type (expression of scale: qualitative/quantitative, verbal/graphical), scale range (number of levels for scale - in the sense of scale discriminant capacity);

- The scale unit: usually, studies on change adopt scales validated in order to discriminate individuals at one occasion but not necessarily in order to discriminate individual differences. Consequently, comparability of original scores does not necessarily produce comparable differences since the same amount of difference may have different meaning at different points of the scale. Assessing comparability of scale unit is particularly important with reference to computing and interpreting difference scores. Scale unit comparability requires

- Adopting metrical or, at least, ordinal scale;

- Observing the shape of scores distribution;

- Assessing change on a metric interval scale (equal level of change at any level of scale).

Nevertheless, adopting categorical scale in social researches cannot be disregarded and need the assessment of scale comparability, even if through a "soft" (not statistical) approach.

Types of measures: comparability has to consider that two different kinds of measures can occur:

- Point measures, obtained for a single point in time; the problem is define the span of time to which assign the point measure;

- Interval measures, involving a count of events, or frequency, measured for an interval of time; these measures are defined in terms of the amount of time over which the measurement is taken.

Nature of data: with reference to the theory of data defined by Coombs (Jacoby 1991), in subjective measurement four different data can be identified:

10 Traditionally, these kinds of data apply to

- Survival and reliability studies, when the observation on an individual begins when the characteristics of interest is first diagnosed;

- Incidence studies, when the duration is measured from the origin (rate of occurrence, obtained by longitudinal follow up); 
- Single stimulus data, produced — for example—by subjects' answers to questions on a rating scale,

- Stimulus comparison data, produced by comparing each individual with a characteristic (subject 'possesses' $x$ units of the characteristic),

- Similarities data, produced by comparing individuals (the degree to which two subjects exhibit the same behavior, level of life satisfaction, and so on),

- Preferential choice data, produced by comparing each individual with a particular stimulus in terms of preference (a subjects likes or prefers a particular activity, sport, and so on).

With reference to repeated data, another classification of type of data can be identified, producing the following categories (Lindsey 1999) ${ }^{10}$ :

- General continuous data: repeated data that take the form of quantitative measures supposed to be any value on the defined continuum; however this assumption can be met only at a high theoretical level; this is particularly true in subjective measurement.

- Categorical and count data: the obtained data represent the number of time a particular event has occurred on the same individual; when such response are distinguished for the same individual by no explanatory variable, events can be aggregated as counts (one category of event is being observed, like a subjective behavior related to quality of life perception); one common use of counts is to measure rates.

- Duration and survival data: the obtained data represents a duration (waiting time to an event). A history is a series of successive events, with the accompanying duration between them; the study of such processes in subjective dimensions requires: (a) a continuous variable measuring the passed time, (b) a discrete variable indicating whether an event has occurred, or not, at each point of time, (c) one or more variables indicating relevant information about the event.

Even if useful, this classification is difficult to be applied to subjective dimension measurement.

Testing comparability with reference to the adopted measurement approach is particularly important in studies on subjective change as well as in trend studies when it could be very dangerous comparing aggregated individual data over time, with regard to a specific characteristic, obtained through different approaches.

In general, meeting comparability conditions is particularly important in studying change of subjective dimensions since we may reach different conclusions on change condition according to different data approaches both in trend (involving group comparisons) and process (involving individual comparisons) approaches. Even if level of comparability seems to be less severe in trend approach, comparing data obtained by different, for example, scaling techniques (even if by same questionnaire) has to consider the different scale performance in discriminating individuals (Maggino 2003).

All these elements (schematically represented in Fig. 7) play a crucial role in testing reliability and validity of measurements over time.

Footnote 10 continued

- Prevalence studies, when the interest is on the frequency in a population at a given point of time (in a fixed population, prevalence $=$ incidence $\mathrm{X}$ duration).

- Survival and incidence are modeled by intensity whereas prevalence by probability. 


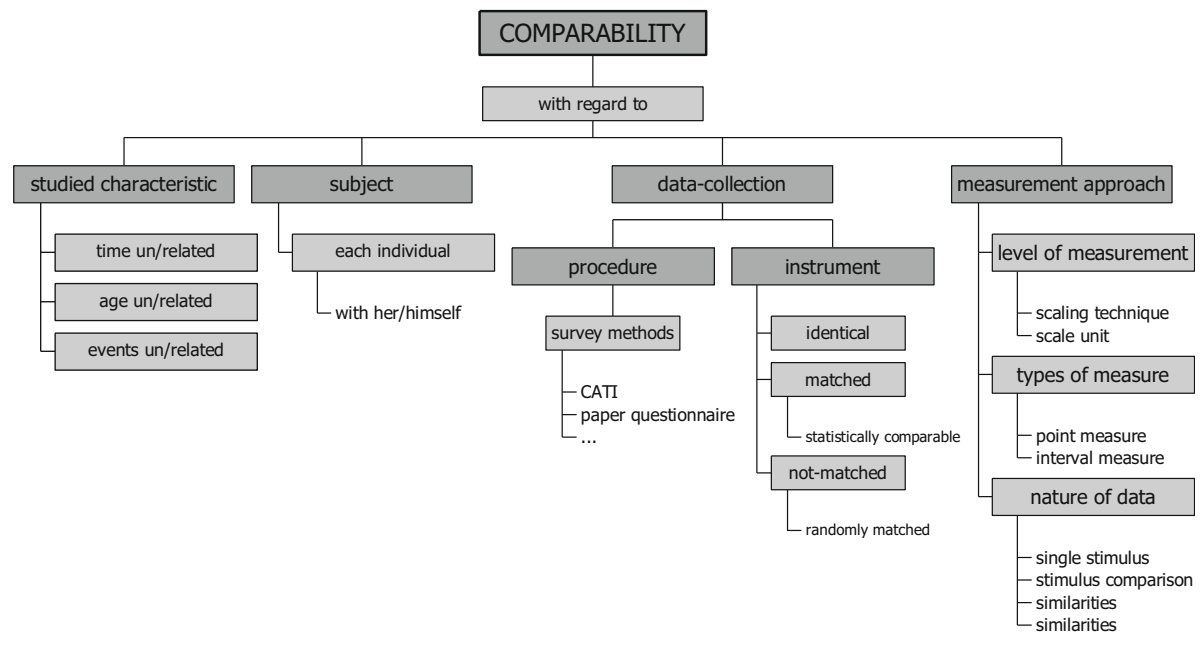

Fig. 7 Elements of comparability to be considered in a study on change

\subsection{Analytical Aspects}

The purpose of data analysis in change studies is to represent observations in order to show regularities of empirical data and to come to valid statement about it.

Data analysis approaches have different characteristics with reference to

- The defined model

- The adopted design

- Measurement approach (continuous, categorical, count and duration data, Lindsey 1999).

\subsubsection{Data Analysis Approaches Related to Model Definition}

By referring to data analysis approaches, we can distinguish between two different models:

- Time-related models, which requires typical probabilistic approaches, such as time series analysis (quantitative approach) and turnover table (qualitative approach);

- Change-in-structure models, for which multivariate approaches exist.

Time-series approach. Time series analysis is typically applied in analysis of dynamic system when many repeated measures are observed. The principal goals of this analysis are: forecast future trends and drawing a possible internal structure of the series (Visser 1985). Time series analysis can also be applied in analysis of individual change. However, especially when each series regards one individual, the approach is difficult to be applied since (Holtzman 1963):

(a) Time variable can bring other uncontrolled variables;

11 If the systematic variance of a given observation depends upon preceding observations the time-series defines a Markoff chain (the order of which depends upon the number of preceding dependence observations). 
(b) Time intervals are arbitrary and, as a result, difficult to allow control of continuous variables;

(c) Repeated observations are not independent producing a serial correlation and making unusable the great part of statistical models. ${ }^{11}$

The analysis of individual change through time-series approach shows further problems regarding statistical inference and missing data.

Mobility/rotation/turnover tables (Chazel et al. 1970; Hout 1983; Hagenaars 1990). When change is observed through categorized data, the analysis needs to adopt different approaches, like the mobility table approach, whose goal is to measure the rotation index instead of line parameter estimates.

Multivariate approach. This approach is applied when the main point of interest is the analysis of consistent changes (in correlation terms) of a set of variables (change-instructure analysis).

An important role in procedure selection is played by the capacity of the procedure to deal with complex data structure like those yielded by repeated measures. In particular, the multivariate analysis of repeated measures requires a three-way data matrix (Visser 1985) in which the three dimensions represent, respectively, occasions, individuals and variables. Multivariate procedures can be distinguished in (Visser 1985) (1) simple descriptive procedures (numerical and graphical techniques), (2) exploratory procedures (aimed at representing changes in structure), (3) confirmatory procedures (aimed at testing specific models).

Many examples of three-mode analysis are available for explorative (three-mode factor analysis or dynamical factor analysis, designed for the analysis of multiple time series) and confirmative factor analysis (Tucker 1963; Harris 1963; Kaiser 1963; Cattell 1963; Bartholomew and Knott 1999) and for multidimensional scaling (Cox and Cox 1994).

The complexity of a three way data matrix can be dimensionally reduced. This can be done by selecting one unit for the analysis, selecting one variable on which conducting the analysis, reducing individual values into one (for each variable), reducing values of several variables into one value (for each individual), flattening out: ${ }^{12}$

Multivariate approach for experimental data: analysis of variance. Analysis of variance allows analysis of experimental data representing characteristics in different and systematically varied conditions at different points of time to be accomplished (Visser 1985). In order to apply this approach, the following assumptions have to be met. ${ }^{13}$

When the interest is on differences between treatment effects (repeated observation experimental design) the reference approach is multivariate analysis of variance (MANOVA); when the interest is also on development of the effects over time, the reference approach is a combination of analysis of variance and regression analysis (Bock 1963).

\footnotetext{
${ }^{12}$ In order to avoid the arbitrary decision involved in the previously presented approaches, yielding arbitrary approximations, the data matrix is sliced in three different direction; each arrangement is a table in which one dimension represents one of the original matrix dimension and the other represents the Cartesian product of the other two dimensions; the resulting three tables are transposed in such a way that the rows may be interpreted as observations and the columns as variables.
}

13 The assumptions concern:

- Experimental conditions: each individual is assumed to be randomly assigned to treatments and to stay in the same treatment group during the experiment;

- Statistical characteristics of data: normal distribution of observed variables and equal variances and covariances between observations over time for all individuals. 


\subsubsection{Data Analysis Approaches}

Data analysis approaches can be mainly distinguished according to the adopted design; particularly, designs allow analyses at two different levels:

- Macro change: analysis of change at group level, allowed by repeated studies;

- Micro change: analysis of change at individual level, allowed only by panel studies.

As described below, each level allows different goals to be accomplished (Engel and Reinecke 1996).

Analysis of macro change: trend analysis. The main goal of analysis of macro change is to detect and to compare trends. The trend is defined as the "change expressed as function of time". Actually, the analysis is aimed at the decomposition of real trends and irregular trends; particularly the time variable can be observed in term of, or, better, the real trend can be decomposed in terms of (Glenn 1977; Menard 1991; Firebaugh 1997):

- Period of observation (for instance "year"), interpretable as "changes over time" effect, that refers to change produced by influenced related to historical age under study;

- Age of observed individuals, interpretable as "life cycle and developmental changes" effect, that refers to change produced by influenced related to age (considered as individual life-cycle status);

- Cohort: each observed individual is member of cohort, defined with reference to particular conditions (geographical area, event, time of particular event, generation, year of birth, and so on) and interpretable in terms of effects. Observed cohort difference could be interpreted with reference to common experiences or reactions of a cohort (according to the cohort definition).

Each of these may represent an explanatory effect of change to be considered (separately or in combination) in the model. Four methods can be identified in order to study group trends (Firebaugh 1997):

1. Trend analysis: analysis of average changes in a group over time and comparison between different trends (coincident, parallel, converging, diverging, crossed trends);

2. Proximate decomposition of trends (proximate source of change), distinguishing between net change among individuals and gross change due to group turnover; the analysis is based upon linear regression approach;

3. Change decomposition of aggregate change in one variable in terms of change in levels and effects of other variables; the analysis is based on decomposition equation regression;

4. Changing-parameter method: analysis of change in effects of variables at individual level, in order to determine the time-dependence of individual-level relationships.

Since repeated studies are based on substantially independent samples, trend analysis provides sufficient description of change but fails in providing empirical explanations of change process.

Analysis of micro change: process analysis. It is not simply a descriptive analysis but also explanatory (process analysis or internal analysis). The analysis, allowed by panel studies, is accomplished at individual-level by investigating covariation over time. By taking into account the fundamental constraints aimed at establishing a causal relationship (covariation, temporal precedence and nonspuriousness), we can observe different kinds of change (Menard 1991), initiation (referring to the first time that a case enters a particular state), 
escalation/reduction (referring to the entry of, respectively, a higher or a lower state, on an ordinal scale), suspension (referring to a permanent or temporary exit from all states that indicate involvement a particular state; this kind of change is not always significantly present).

Collecting data in order to study and analyze processes described by this model may be difficult because of possible long terms occurring. The problem, called 'left-hand censoring', indicates the failure to detect a change because it happened before the period of data collection.

Consequently in order to unravel causal relationships, it is important to qualify the model in terms of adequateness of time lag (that is, interval between data collection periods of time) in order to allow

- Change to be detected in a variable clearly separated from change in another ${ }^{14}$

- An effect to be produced by occurrence of cause.

\subsection{From Measuring Change to Forecasts: Criticisms}

Understanding the theoretical, methodological and analytical aspects in observing change allows any prediction and forecast to be correctly interpreted and considered.

Actually, measuring change may have different implications and turn out to be also a useful tool for forecasting in the perspective of other exercises, like that aimed at modeling sustainability of our societies (e.g., modeling relationship between hope, trust and identity through the catastrophe theory). In this sense, managing the "change" logic represents a good starting point for policies. It could be very interesting to analyse how many times studies on change led the researchers to real serendipities (Merton and Barber 1992).

In order to define possible future scenarios, modeling the available knowledge (theoretical and/or practical) around the phenomenon in the right and correct way represents a crucial issue. This is particularly true when adopting measuring change as the logical perspective allowing and supporting the forecasting exercise to be pursued.

However, many issues make the forecasting exercise very difficult to manage especially in complex contexts like those describing quality of life and wellbeing, which require different levels to be considered (individual, community, environmental). Among those difficult issues, it should be considered that, according to the classic scientific explanation, the result of any prevision can be (1) assessed and explained only ex post, ${ }^{15}$ and (2) influenced by the prevision in itself.

Credibility and success of predictions depend upon the capacity of identifying the elements characterizing a certain reality and allowing its complexity to be modeled.

${ }^{14}$ If the change in both variables occurs in the same period, there are different possible explanations (Menard 1991):

(a) The two variable measure the same thing,

(b) The two variables are spuriously related, having a common cause producing changes in both,

(c) The length of measurement period does not allow to separate the two changes.

15 The debate on the relationship between explanation and forecasting (some authors use the term "prediction", others use "prevision") is an important part of the wider debate on scientific explanation which started from a paper by Hempel and Oppenheim (1948), considered the foundation of scientific explanation view. Among the authors who discussed the topic of forecasting in this context, it should be cited Scriven (1959), Goodman (1959), Nagel (1961), Hempel (1965, 1977), Salmon (1971), and Coffa (1974). For a very interesting history of the debate on scientific explanation, see Salmon (Salmon 1989). 
Actually, the level of knowledge and complexity of the reality may cause any effort in formulating credible predictions to be fruitless and useless. By thinking at quality of life and the use of the change logic, the elements, which can undermine credible and useful predictions, are:

- Overestimating the change in terms of tendencies, cadences and trends,

- Underestimating the impact that any change can have on the reality,

- Overestimating past experiences,

- The presence of complex causal systems,

- The presence of particular elements which cannot be exportable and projectable.

That is because in order to face the delicate topic of prediction, it is necessary identifying different approaches that, realistically, are not strictly aimed at forecasting but allow multiple possible futures to be obtained by applying an iterative process and involving different experts' opinions.

In particular, to overcome the limits of the traditional approaches to prediction, it could be useful to adopt alternative approaches like "scenarios analysis" ${ }^{16}$ which is particularly useful in the presence, like it happens in the quality-of-life field, of (1) phenomena with high levels of complexity and uncertainty (2) events with low possibility but big impacts, and (3) context related to different views and opinions (policy level).

However, the risk in adopting alternative approaches is to launch in defining scenarios, which turn out to be less plausible than utopian, yielded by wishes and attracting images.

\section{A Possible Approach to Forecasting Through Complexity: The Futures Studies}

\subsection{An Epistemological Puzzle}

After getting aware of the paramount importance of analyzing change for understanding possible evolutions of the future, the social researcher must, therefore, face a very important epistemological problem: can a social scientist make social previsions without leaving the scientific context? Does talking about "prediction" or "forecasting" mean necessarily leaving science? The answer is not necessarily univocal. In order to move around this arduous epistemological obstacle and to solve this intriguing meta-scientific puzzle, Futures Studies (FS) can and may represent an interesting solution.

The main epistemic principle of FS is that the future is not just one, because it can have many developments. The mission of FS is to promote social policies and actions for the future well-being of societies without "colonizing" the future. For this reason, they insist on using the plural "futures". FS do not define themselves as a proper science but mainly as a scientific discipline (Ammassari 1969; Barbieri Masini 1986, 1993, 1994, 2000; Lo Presti 1998; Bell 2003, 2004; Rizza 2003; Facioni 2012).

\footnotetext{
${ }^{16}$ Generally, the scenario analysis considers the following stages: (1) identifying critical and external factors (social, technological, economic, environmental, and political); (2) identifying alternative futures (forecasts); (3) developing strategies (decisions). The first stage represents the crucial step in defining admissible scenarios.

17 One of the most important theorists of FS was Bertrand de Jouvenel, philosopher who-in the Fifties of XX century-founded the periodical Futuribles and published L'art de la conjecture in 1964: in this work, he theorized the difference between desirables (considered as utopia), plausible, probable, and possible
} 
Although their research object is situated in the future (or rather, in futures), the FS base their assertions on elements related to the specific context of present time. The scientificity of FS lies in the accuracy of the methods used in current time to build data (Campelli 1994) collected at present time. The non-scientificity of FS lies in their own object's collocation in time only (reaching well-being in futures). The non-science status allows FS to imagine and experiment more than in the proper science context itself. For this reason, FS can provide precious suggestions to the scientific context as a whole-it also because of their natural vocation to inter-disciplinary and trans-disciplinary approaches. ${ }^{17}$ Although FS researchers may also draw inspiration from utopias, they can be considered a particular kind of social change analysts.

Making FS only superficially may seem a kind of planning (which is a mono-directional way of deciding on the future). Actually, they are something very different (and difficult). The choices made in the ambit of FS are based on both qualitative and quantitative data, ${ }^{18}$ whose sources are reliable (e.g., official statistics, experts' opinions on the evolution of social phenomena, etc.). By referring to social sciences as a whole, even though FS have a generic vocation to interdisciplinary approach, their real "methodological vocation" is the trans-disciplinary approach (Somerville and Rapport 2000). The distinction between the two perspectives is marked: unlike interdisciplinary perspective, trans-disciplinary approach is aimed at overcoming the boundaries between disciplines, in search of a language, tools and even goals and issues. Applying trans-disciplinary approach represents certainly a fascinating job but also a controversial methodological issue since it aims at reconciling different conceptualizations from various disciplines. For example, the meaning related to different periods (short/medium/long term) may be conceived differently in sociology, demography, or economics. Overcoming the differences requires a trans-disciplinary effort.

Footnote 17 continued

futures (de Jouvenel 1964). Gaston Berger, another important French theorist, stressed the importance of the link between policy makers and FS and theorized the politically active FS.

American theorists gave a great contribution to the discipline by developing specific techniques (scenarios, Delphi, simulation systems) especially thanks to the RAND Corporation contribution.

Also Italy gave important contribution to FS through prestigious personalities like Aurelio Peccei (former FIAT manager and founder of the Club of Rome), Pietro Ferraro (industrial who, inspired by the French Futuribles, founded the Italian journal Futuribili), Eleonora Barbieri Masini (sociologist who coordinated the World Futures Studies Federation for years and very important scientific researches based on Futures Studies, such as "Household, Gender, and Age", which will described further below).

Beyond the nationality of those who contributed to the discipline, FS have indeed the natural vocation to interconnect countries and people. For example, the Club of Rome included members from Russia and America (in spite of the Cold War).

The Club of Rome's report The Limits to Growth, developed by M.I.T. scientists (Meadows et al. 1972) was indeed one of the most debated scientific report on environment risk of all times. It is clearly a nontrivial approach to social research indeed: the underlying issues are complex and involve many themes related to methodology of social sciences.

${ }^{18}$ Sections 1 and 2 describe many of the statistical techniques FS utilize. Furthermore, the discipline developed also its own techniques: the most well-known are with no doubt Delphi Method and scenarios.

${ }^{19}$ According to Weber, the scientists must explain their ethical and political beliefs and their opinions; at the same time, these value-aspects must never influence, in any way, their scientific work. The valuefreedom can be a very faint boundary even in average social research: a thin line clearly crossed by FS researchers. Die Obiectivität socialwissenschaftlicher und sozialpolitischer Erkenntnis (Weber 1904) and Wissenschaft als beruf (1917-1919). 


\subsection{Theoretical and Methodological Issues}

FS aim at building better future perspectives by taking into account and analysing the emerging critical issues as well as the emerging positive elements in the present time.

From the theoretical point of view, the FS recall the principle of value-freedom (freedom from value judgment) of the scientist (as theorized by Weber). ${ }^{19}$ Consequently, FS researcher's education and values may have a clear influence on their work. This is because FS need to ground on values which have to be shared accepted through all cultures, so as they can work on widely shared goals, leading secure benefits to future generations.

\subsection{Futures Studies and the Social Research on Futures}

\subsubsection{Social Knowledge, Studying Change, Social Research Methodology and Social Indicators: Two Recent Examples of the Futures Studies Approach}

The FS approach is not only a useful exercise of democracy, or a very interesting outlook on social sciences, actually it can have a solid impact on the citizen's quality of life.

Moreover, even though the research is focused only on present time, the acquired knowledge impacts almost certainly, through the subsequent [policy] action, on the futures. ${ }^{20}$ In this perspective, researchers, as well as policy makers, have to take into account that the conceptualization of quality of life can change over time (Orsini and Montecolle 2012). This issue seems to become crucial in the delicate role that official statistics have in any society. A meaningful sign of this clear sensitivity of official statistics to futures was the slogan of the XI Italian Conference on Statistics: "Understanding the present time for planning the future" (February 2013). Also the Italian Equitable and Sustainable Well-being project (Benessere Equo e Sostenibile-BES) aimed at identifying new indicators of wellbeing supporting policy decision at country level, as results from the cooperation of the Italian National Institute of Statistics (ISTAT) and the National Council for Economics and Labour (CNEL), represents also a clear sign of a remarkable sensibility to the future issue. The project identified twelve key domains where wellbeing has to be observed and monitored: health, education and training, work and life balance, economic well-being, social relationships, politics and institutions, security, subjective well-being, landscape and cultural heritage, environment, research and innovation, and quality of services. $^{21}$ The project is actually constructing an instrument for policy decision, which means that is not strictly devoted to describing the present but to "constructing" the future by looking also at not only wellbeing but also its fair distribution and sustainable promotion: the selected indicators, by allowing the observation and monitor of changes, urge what is actually a future programme in the perspective highlighted by FS. In other words, this project achieves the link between quality of life studies, FS and official statistics, a logic and pragmatic link, carried out by the best practices in social research. Although the B.E.S. Report represents a wide-ranging vision, a Futures Studies approach can be applied

\footnotetext{
${ }^{20}$ For an analysis of subjective well-being indicators, see: http://www.fqts.org/dati/doc/128/doc/191.pdf.

21 The identified indicators include also subjective data able to picture reality also in this perspective. ISTAT has a long experience in collecting subjective data. The main source of ISTAT'S subjective data is represented by the Multipurpose Surveys System, which provide that Annual Survey on Aspects of Everyday Life.
} 
(with the official statistics back-up) also in order to solve a social problem (by introducing a change, actually).

In November 2013 started in Italy the social campaign "Recognize the violence", aimed to avoid the violence against women in Italy and held on the occasion of the International Day for the Elimination of Violence against Women, promoted by the UN ( 25 November). It included billboards all over the country, the presence in national newspapers and the periodical press and widespread dissemination on the web.

In spite of the constant decline of the value of the homicides rate in Italy, the percentages of women murdered by their partners (or ex-partners) are remained almost the same over the years (ISTAT 2013). This needs a particular action able to impact on a cultural factor. However, any progress in cultural terms takes long time to be effective.

Since the problem needs to find an urgent solution, i.e., it is urgent to prevent violence against women, a social complain has been launched by the government. The campaign was accomplished by applying a FS approach. The trans-disciplinary action adopted put together communication techniques, findings from statistical data, and psychology. Since addressing violent men was considered useless; the message of the social campaign were only addressed to women. One of the slogans ("you have just one way to change your violent boyfriend: replace your boyfriend with another one") reflected official data which refer that women who suffer violence from a men remain with their partners hoping that he will change his attitudes. Again, consistently with official data (ISTAT 2007) referring that violent people often were present at violence as children, another message of the campaign was "Don't marry a violent man: children learn quickly".

\section{Conclusions}

Modeling forecasts in the field of quality of life means to:

- Take into account different issues, like past events and decisions, current situation and trends, forces (e.g., policy decisions) driving change, situations allowing or barring change, continuities and discontinuities in actions;

- Look at future in terms of possibilities (expressed as probabilities or un/certainties) and in terms of impacts of future events (explored in terms of severity, likelihood and timing).

In this perspective, social research on change has a solid methodological apparatus which, however, can have some limit in predicting and figuring out future trends.

As we have seen, predicting change values and trends is not possible simply by observing those obtained in the past, because of the complexity of the observed phenomena mainly due to (1) the dimensionality of phenomenon; (2) the relationships between different aspects of the phenomenon (linear and non-linear); (3) the causality, which could be direct or indirect; and (4) the interpretation of the entity of change (e.g., small change can have great impact); (5) the perspective of observation, which can be internal/external and local/global.

The critical issues of the forecasting exercise are several, also interrelated.

The theoretic perspective is particularly important in order to formulate models. However, models formulation shows a risk represented by the attraction to simplify the line of reasoning especially if also by resting on experiences (i.e., "if in the past after event A, event B occurred, then we can assert that when event A will occur in the future, even B will 
occur as well"). The validity of this line of reasoning depend upon different issues: (a) in which way event A and event B have been defined and measured, (b) how many times the sequence of events occurred in the past (event B after event A), (c) if the description of the phenomenon composed by event $\mathrm{A}$ and event $\mathrm{B}$ is valid also in the future.

Another critical issue is represented by the choice of indicators which can indeed determine the correctness of the analysis, as well as its failure.

These, as well as others, critical issues suggest that in order to manage the prediction issue in the field of quality of life research, a composite structure should be defined linking social research methodology on change to the Social Indicators approach and the Future Studies perspective.

As seen, the logic of social research is also applied to para-scientific approaches such as the Futures Studies. A correct methodological approach is necessary indeed for anyone concerned with forecasting, because of the possible consequences of an incorrect forecasting. This reminds us to very important scientific topics like the risk underlying the cognitive biases (Kahneman and Tversky 1973), as well as the complexity of interpretation models related to social sciences (Lazarsfeld 1946). These issues, however, refer to a single, fundamental and inescapable topic: the responsibility of the scientific community on the welfare of society-including the future societies' one.

\section{References}

Ammassari, P. (1969). Della previsione nelle scienze sociali: il problema ricorrente, in "Futuribili" n. 16. Roma Editrice Futuribili.

Barbieri Masini, E. (1986). La previsione umana e sociale. Roma: Editrice Pontificia Università Gregoriana. Barbieri Masini, E. (1993). Why futures studies?. London: Grey Seal.

Barbieri Masini, E. (1994). La previsione. Idee, protagonisti, nodi problematici, "Futuribili" n. 1. Milano, Franco Angeli.

Barbieri Masini, E. (2000). Penser le Futur-L'essentiel de la prospective et de ses méthodes. Paris: Dunod.

Bartholomew, D. J. (1996). The statistical approach to social measurement. San Diego, London: Academic Press.

Bartholomew, D. J., \& Knott, M. (1999). Latent variable models and factor analysis. London, Sydney, Auckland: Arnold.

Bell, W. (2003). Foundations of futures studies. History, purposes, and knowledge. New Brunswick, London: Transaction Publishers.

Bell, W. (2004). Foundations of futures studies. Values, objectivity, and the good society. New Brunswick, London: Transaction Publishers.

Bock, R. D. (1963). Multivariate analysis of variance of repeated measurements. In C. W. Harris (Ed.), Problem in measuring change. Madison: University of Wisconsin Press.

Brown, C. (1995). Chaos and catastrophe theories, Sage University Paper Series on Quantitative Applications in the Social Sciences, series no. 07-107. Newbury Park, CA: Sage.

Bryk, A. S., \& Raudenbush, S. W. (1992). Hierarchical linear models: Applications and data analysis methods, advanced quantitative techniques in the social sciences series, Vol. 1, Thousand Oaks, London, New Delhi: SAGE Publications.

Campbell, D. T. (1963). From description to experimentation: Interpreting trends as quasi-experiments. In C. W. Harris (Ed.), Problem in measuring change. Madison: University of Wisconsin Press.

Campelli, E. (1994). Il metodo e il suo contrario. Franco Angeli: Milan.

Cattell, R. B. (1963). The structuring of change by P-technique and incremental R-technique. In C. W. Harris (Ed.), Problem in measuring change. Madison: University of Wisconsin Press.

Chazel, F., Boudon, R., \& Lazarsfeld, P. (1970). L'analyse des processus sociaux, collection Méthodes de la sociologie. Mouton, Paris: La Haye.

Coffa, J. A. (1974). Hempel's ambiguity. Synthese, 28(2), 141-163.

Cox, T. F., \& Cox, M. A. A. (1994). Multidimensional scaling. London: Chapman \& Hall.

De Jouvenel, B. (1964). L'art de la conjecture. Monaco, Éditions du Rocher: Futuribles. 
Embretson, S. E. (1994). comparing changes between groups: Some perplexities arising from psychometrics. In D. Laveault, B. D. Zumbo, M. E. Gessaroli, \& M. W. Boss (Eds.), Modern theories of measurement: Problems and issues. Ottawa: Edumetrics Research Group, University of Ottawa.

Engel, U., \& Reinecke, J. (1996). Analysis of change. Berlin, New York: Walter de Gruyter.

Facioni, C. (2012). Il contributo italiano ai Futures Studies. http://padis.uniroma1.it/.

Firebaugh, G. (1997). Analyzing repeated surveys, Sage University Paper Series on Quantitative Applications in the Social Sciences, series no. 07-115. Newbury Park, CA: Sage.

Glenn, N. D. (1977). Cohort analysis, Sage University Paper Series on Quantitative Applications in the Social Sciences, series no. 07-005. Newbury Park, CA: Sage.

Goldstein, H. (1979). The design and analysis of longitudinal studies. London: Academic Press Inc., Ltd.

Goodman, N. (1959). Fact, fiction and forecast. Cambridge: Harvard University Press.

Hagenaars, J. A. (1990). Categorical longitudinal data. Newbury Park, CA: Sage.

Harris, C. W. (1963). Canonical factor models for the description of change. In C. W. Harris (Ed.), Problem in measuring change. Madison: University of Wisconsin Press.

Hempel, C. G. (1965). Aspects of scientific explanation. Florence: The Free Press, A Division of the MacMillan Company.

Hempel, C. G. (1977). Nachwort. Berlin: Walter De Gruyter.

Hempel, C. G., Oppenheim, P. (1948). Studies in the logic of explanation. In: Philosophy of science (n. 2, pp. 135-175). University of Chicago Press.

Holtzman, W. H. (1963). Statistical models for the study of change in the single case. In C. W. Harris (Ed.), Problem in measuring change. Madison: University of Wisconsin Press.

Hout, M. (1983). Mobility tables, Sage University Paper Series on Quantitative Applications in the Social Sciences, series no. 07-031. Beverly Hills and London, CA: Sage.

Huckfeldt, R. R., Kohfeld, C. W., \& Likens, T. W. (1982). Dynamic modelling. An introduction, Sage University Paper Series on Quantitative Applications in the Social Sciences, series no. 07-027. Beverly Hills, CA: Sage.

ISTAT. (2007). La violenza e i maltrattamenti contro le donne dentro e fuori la famiglia. Anno 2006. http:// www.istat.it.

ISTAT, CNEL, B.E.S. (2013). Il benessere equo e sostenibile in Italia, Roma. http://www.istat.it.

Jacoby, W. G. (1991). Data theory and dimensional analysis, Sage University Paper Series on Quantitative Applications in the Social Sciences, series no. 07-078. Newbury Park, CA: Sage.

Kahneman, D., \& Tversky, A. (1973). On the psychology of prediction. Psychological Review, 80(4), 237-251.

Kaiser, H. F. (1963). Image analysis. In C. W. Harris (Ed.), Problem in measuring change. Madison: University of Wisconsin Press.

Lazarsfeld, P. F. (1946). Interpretation of statistical relations as a research operation. In P. F. Lazarsfeld \& M. Rosenberg (Eds.) (1955), The language of social research (pp. 115-125). The Free Press.

Lazarsfeld, P. F., \& Henry, N. W. (1968). Latent structure analysis. Boston: Houghton M. Company.

Lindsey, J. K. (1999). Models for repeated measurement (Vol. 2). Oxford: Oxford University Press.

Lo Presti, A. (1998). Previsioni sociologiche e Futures Studies: un tentativo di ricomposizione logica e concettuale, in "Sociologia e ricerca sociale" n. 55. Milano, Franco Angeli.

Maggino, F., \& Schifini D’Andrea, S. (2003). Different scales for different survey methods: Validation in measuring quality of university life. In M. J. Sirgy, D. Rahtz, \& J. Samli (Eds.), Advances in quality of life research. Dordrecht: Kluwer Academic Publisher.

Markus, G. B. (1979). Analyzing panel data, Sage University Paper Series on Quantitative Applications in the Social Sciences, series no. 07-018. Beverly Hills and London, CA: Sage.

Meadows, D. H., Meadows, D. L., Randers, J., \& Behrens, W. W. (1972). The limits to growth: A report for the club of rome's project on the predicament of mankind. Geneva: Club of Rome.

Menard, S. (1991). Longitudinal research, Sage University Paper Series on Quantitative Applications in the Social Sciences, series no. 07-076. Newbury Park, CA: Sage.

Merton, R. K., \& Barber, E. G. (1992). The travels and adventures of serendipity. A study in historical semantics and the sociology of science. Princeton: The Princeton University Press.

Nagel, E. (1961). The structure of science: Problems in the logic of scientific explanation, Harcourt. New York: Brace \& World.

Orsini, S., \& Montecolle, S. (2012). Satisfied or dissatisfied? An analysis of the results of "aspects of daily life" Italian survey on households. Social Indicators Research, 48, 115-133.

Rizza, S. (2003). Il presente del futuro. Milano, Franco Angeli: Sociologia e previsione sociale.

Rogosa, D. R., Brand, D., \& Zimowski, M. (1982). A growth curve approach to the measurement of change. Psychological Bulletin, 90, 726-748. 
Rogosa, D. R., \& Willett, B. (1985). Understanding correlates of change by modeling individual differences in growth. Psychometrica, 50, 203-228.

Salmon, W. C. (1971). Statistical explanation and statistical relevance. Pittsburg: University of Pittsburg Press.

Salmon, W. C. (1989). Four decades of scientific explanation. Minneapolis: Regents of University of Minnesota.

Scriven, M. (1959). Explanation and prediction in evolutionary theory. Science, 130(3374), 477-482.

Somerville, M. A., \& Rapport, D. J. (2000). TRANSDISCIPLINARITY: Recreating integrated knowledge. Oxford: EOLSS Publishers Co. Ltd.

Tucker, L. R. (1963). Implications of factor analysis of three-way matrices for measurement of change. In C. W. Harris (Ed.), Problem in measuring change. Madison: University of Wisconsin Press.

Visser, R. A. (1985). Analysis of longitudinal data in behavioural and social research. Leiden: DSWO Press.

Weber, M. (1904). Die Obiektivität sozialwissenschaft licher und sozialpolitischer Erkenntnis in Archiv für Sozialwissenschaft und Sozialpolitik, XIX, pp. 22-87

Webster, H., \& Bereiter, C. (1963). The reliability of change measured by mental test scores. In C. W. Harris (Ed.), Problem in measuring change. Madison: University of Wisconsin Press. 